\title{
Article \\ Structural Analysis of Botulinum Neurotoxins Type B and E by Cryo-EM
}

\author{
Sara Košenina ${ }^{1, \dagger}{ }^{,}$Markel Martínez-Carranza ${ }^{1, \dagger}$, Jonathan R. Davies ${ }^{1}$, Geoffrey Masuyer ${ }^{1,2, *(1)}$ \\ and Pål Stenmark 1,3,* (D) \\ 1 Department of Biochemistry and Biophysics, Stockholm University, 10691 Stockholm, Sweden; \\ sara.kosenina@dbb.su.se (S.K.); markel.martinez-carranza@pasteur.fr (M.M.-C.); \\ jonathanrd@gmail.com (J.R.D.) \\ 2 Centre for Therapeutic Innovation, Department of Pharmacy and Pharmacology, University of Bath, \\ Bath BA2 7AY, UK \\ 3 Department of Experimental Medical Science, Lund University, 22184 Lund, Sweden \\ * Correspondence: geoffrey.masuyer@dbb.su.se (G.M.); stenmark@dbb.su.se (P.S.) \\ + These authors contributed equally to this work.
}

Citation: Košenina, S.; MartínezCarranza, M.; Davies, J.R.; Masuyer, G.; Stenmark, P. Structural Analysis of Botulinum Neurotoxins Type B and E by Cryo-EM. Toxins 2022, 14, 14. https://doi.org/10.3390/ toxins 14010014

Received: 6 December 2021

Accepted: 21 December 2021

Published: 23 December 2021

Publisher's Note: MDPI stays neutral with regard to jurisdictional claims in published maps and institutional affiliations.

Copyright: (c) 2021 by the authors Licensee MDPI, Basel, Switzerland. This article is an open access article distributed under the terms and conditions of the Creative Commons Attribution (CC BY) license (https:// creativecommons.org/licenses/by/ $4.0 /)$.

\begin{abstract}
Botulinum neurotoxins (BoNTs) are the causative agents of a potentially lethal paralytic disease targeting cholinergic nerve terminals. Multiple BoNT serotypes exist, with types A, B and E being the main cause of human botulism. Their extreme toxicity has been exploited for cosmetic and therapeutic uses to treat a wide range of neuromuscular disorders. Although naturally occurring BoNT types share a common end effect, their activity varies significantly based on the neuronal cell-surface receptors and intracellular SNARE substrates they target. These properties are the result of structural variations that have traditionally been studied using biophysical methods such as $X$-ray crystallography. Here, we determined the first structures of botulinum neurotoxins using single-particle cryogenic electron microscopy. The maps obtained at 3.6 and $3.7 \AA$ for BoNT/B and /E, respectively, highlight the subtle structural dynamism between domains, and of the binding domain in particular. This study demonstrates how the recent advances made in the field of singleparticle electron microscopy can be applied to bacterial toxins of clinical relevance and the botulinum neurotoxin family in particular.
\end{abstract}

Keywords: Clostridium botulinum; botulism; botulinum neurotoxin; BoNT/B; BoNT/E; cryo-EM

Key Contribution: Botulinum neurotoxins type B and E can cause human botulism and are clinically relevant alternatives to BoNT/A for therapeutic applications. Single particle cryo-EM shows the structural dynamism of these holotoxins, providing an alternative to X-ray crystallography for atomic resolution studies on this toxin family.

\section{Introduction}

The botulinum neurotoxins (BoNT) are potent bacterial toxins produced mainly by toxigenic Clostridium botulinum species [1]. Recently, several new BoNT and BoNT-like proteins have been discovered, considerably expanding this family of proteins beyond their traditional serotype classification [1,2]. The holotoxins are expressed as single-chain proteins that need to be proteolytically activated into a functional di-chain form, which consists of a $\sim 50 \mathrm{kDa}$ light chain (LC) linked by a single disulphide bond to the $\sim 100 \mathrm{kDa}$ heavy chain (HC) [3]. Although they all share a common modular architecture based on the three functional domains necessary for their activity, namely the binding $\left(\mathrm{H}_{\mathrm{C}}\right)$ and translocation domains $\left(\mathrm{H}_{\mathrm{N}}\right)$, which form $\mathrm{HC}$ and the catalytic (LC) domain, they present distinctive properties. In particular, variation in receptor recognition and substrate specificity dictate their species selectivity and pharmacological characteristics [4]. 
Serotypes A, B and E are the main BoNT associated with human botulism [5]. These neurotoxins specifically target presynaptic motoneurons via a dual-receptor binding mechanism, which involves membrane-anchored gangliosides [6-8] and a protein receptor [9]. $\mathrm{BoNT} / \mathrm{A}, / \mathrm{D}, / \mathrm{E}$ and /F recognise the synaptic vesicle glycoprotein 2 (SV2), whereas $\mathrm{BoNT} / \mathrm{B}, / \mathrm{G}$ and /DC utilise one of the synaptotagmin isoforms [10-17]. Receptormediated endocytosis allow neuronal uptake via vesicular compartments where the acidic $\mathrm{pH}$ promotes translocation of the LC into the cytosol [18]. There, LC which is a zinc-protease, can degrade one of the soluble NSF attachment protein receptors (SNARE) responsible for exocytosis [19]. BoNT/A and /E cleave SNAP-25 (synaptosomal-associated protein of $25 \mathrm{kDa}$ ), BoNT/C targets both SNAP-25 and syntaxin, while BoNT/B, /D, /F, /G and /X degrade VAMP isoforms (vesicle-associated membrane protein), resulting in inhibition of neurotransmission and its associated flaccid paralysis [2,20].

Because of their extreme potency, BoNTs have also become the therapeutic molecules of choice for an increasing number of neuromuscular disorders, which include spasticity and dystonia, as well as other chronic conditions such as excessive sweating and migraines [21,22]. Two serotypes, BoNT/A (e.g., onabotulinumtoxinA, abobotulinumtoxinA) and /B (rimabotulinumtoxinB) are currently on the market and approved for therapeutic or cosmetic use. In addition, the variation in activity between natural BoNT serotypes translates into significantly different pharmacological profiles [23]; therefore, other BoNTs are being investigated as alternatives to currently available commercial products. For example, serotype E presents a faster onset of action and a shorter duration of action in humans [24], which may be beneficial for certain clinical applications that have been examined in several clinical trials $[25,26]$.

BoNT/B and /E share approximately $40 \%$ sequence identity with BoNT/A, while homology can vary between 30 to $40 \%$ across other serotypes [3] (Figure 1). The first X-ray crystal structure of a BoNT holotoxin was that of type A [27], followed by BoNT/B [28] and /E [29]. Where the individual domains present identical folds, the overall architecture can vary between a linear configuration of the three functional domains, as seen in BoNT/A and /B, or the 'closed' arrangement seen in BoNT/E, where all three domains interact with each other in a conformation that is also associated with a faster translocation rate $[30,31]$. The structure of many of the single domains has been resolved by X-ray crystallography [3]. However, the full-length holotoxins remain challenging molecules to study with this method (Table 1), and so far, only low-resolution images, although informative, have been reported from single-particle microscopy [31]. The variation in interactions observed between domains and their effects on the toxins' activity suggests that the toxins may present significant flexibility that is important to investigate. For example, structural studies on the closely related tetanus toxin demonstrated $\mathrm{pH}$-mediated domain dynamics that are essential for its transport to the central nervous system and, thus, toxicity [32].

Table 1. Overview of structural information available on clostridial neurotoxins.

\begin{tabular}{|c|c|c|c|c|c|}
\hline & LC & $\mathbf{L H}_{\mathbf{N}}$ & $\mathbf{H}_{\mathrm{C}}$ & Full-Length & BoNT-NTNH \\
\hline BoNT/A & $1 \mathrm{XTF}$ & 2W2D & 2VU9 & 3BTA & $3 \mathrm{~V} 0 \mathrm{~B}$ \\
\hline BoNT/B & 2ETF & 2XHL & $1 \mathrm{ZOH}$ & 1EPW & - \\
\hline BoNT/C & 1QN0 & - & $3 N 7 K$ & - & - \\
\hline BoNT/CD & $(=C)$ & - & 3PME & - & - \\
\hline BoNT/D & $2 \mathrm{FPQ}$ & $5 B Q N$ & 3OGG,3N7J,3OBR & - & - \\
\hline BoNT/DC & $(=D)$ & $(=\bar{D})$ & $3 \mathrm{AZW}$ & - & - \\
\hline BoNT/E & 1T3A & 7K7Y & 7OVW & 3FFZ & $4 \mathrm{ZKT}$ \\
\hline BoNT/F & $2 \mathrm{~A} 8 \mathrm{~A}$ & - & 3FUQ & - & - \\
\hline BoNT/G & 1ZB7 & - & 3MPP,2VXR & - & - \\
\hline BoNT/HA & 6BVD & - & $5 \mathrm{~V} 38$ & - & - \\
\hline BoNT/X & $6 \mathrm{~F} 47$ & - & - & - & - \\
\hline TeNT & 1YVG & - & 1AF9 & $5 \mathrm{~N} 0 \mathrm{~B}$ & $\mathrm{~N} / \mathrm{A}$ \\
\hline
\end{tabular}

Nonexhaustive list of Protein Data Bank records, first-released entries included in this table. Brackets indicate when a domain is similar to another serotype in mosaic toxins. 
a

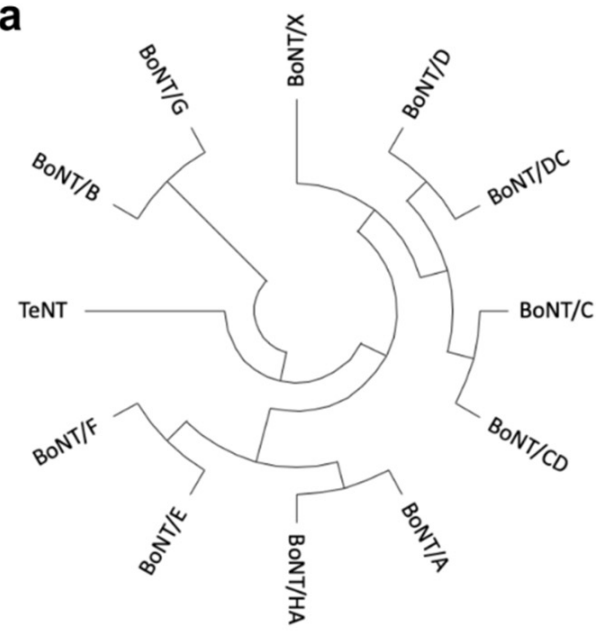

b

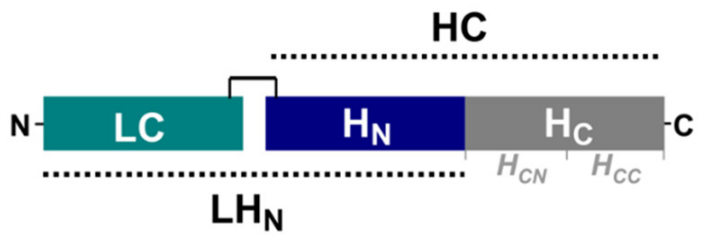

Figure 1. (a) Phylogenetic tree of clostridial neurotoxins prepared with MEGAX [33] from protein sequences aligned with CLUSTALO [34]. (b) Schematic representation of the botulinum neurotoxin domain structure. The toxins are expressed as single-chain proteins but are later proteolytically converted to their active di-chain form, where LC is linked by a single disulphide bridge to HC. The $\mathrm{LH}_{\mathrm{N}}$ fragment has been defined previously as the combined LC $+\mathrm{H}_{\mathrm{N}}$ domains [35].

Here, we present the first cryo-EM structures of BoNT/B and /E by single-particle analysis. The fast development of this technique has made the study of BoNT in near atomic details more feasible. Our results show the subtle local dynamics within the toxins structure, which help us understand their complex mechanism of action. This study demonstrates that cryo-EM is a method of choice to pursue the structural characterisation of full-length botulinum neurotoxins.

\section{Results and Discussion}

\subsection{Single Particle Analysis by Cryo-Electron Microscopy}

\subsubsection{Data Collection and Analysis}

Botulinum neurotoxins consisting of catalytically inactive variants with a poly-histidine tag were recombinantly produced in E. coli and purified to homogeneity using traditional chromatography methods. For each sample, multiple conditions were screened with particle distribution appearing constantly most suitable on glow-discharged holey carbon copper grids. Complete movies were collected on a Titan Krios microscope, resulting in EM maps at 3.6 and 3.7 $\AA$ average resolution for BoNT/B and BoNT/E, respectively, although local resolution varied significantly (Supplementary Figures S1 and S2). The map for BoNT / B appeared overall more precise than that of BoNT/E (Figure 2), even though image processing showed a slight preferential orientation for BoNT/B, which may partially explain the variation in local resolution. For both toxins, particle sizes were consistent with monomers, and secondary structure could clearly be distinguished in the 2D average classification, so that typical features of the individual domains were identifiable. Noticeably, the map quality around the binding domain was generally weaker compared to the rest of the toxin. 

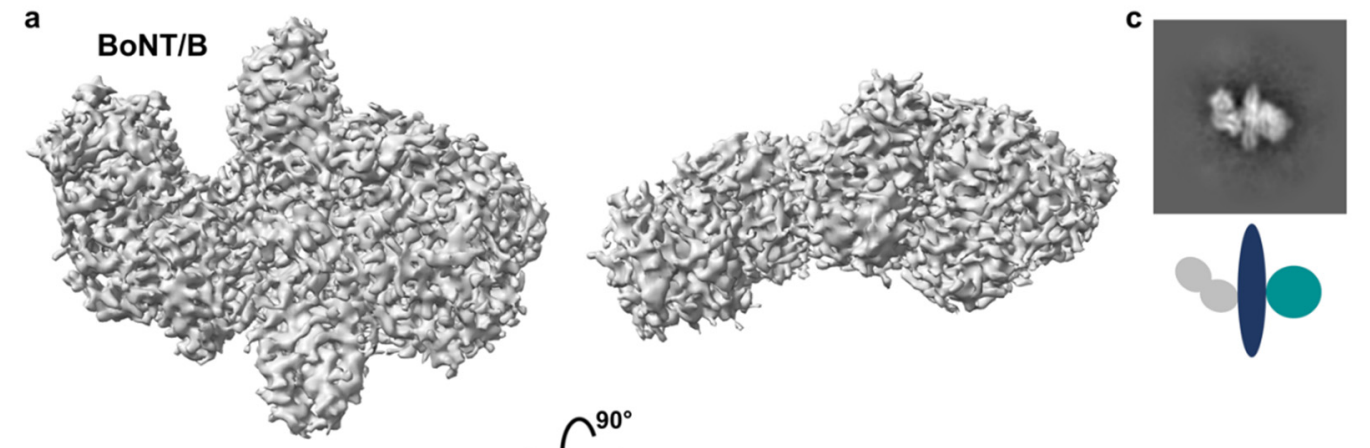

b
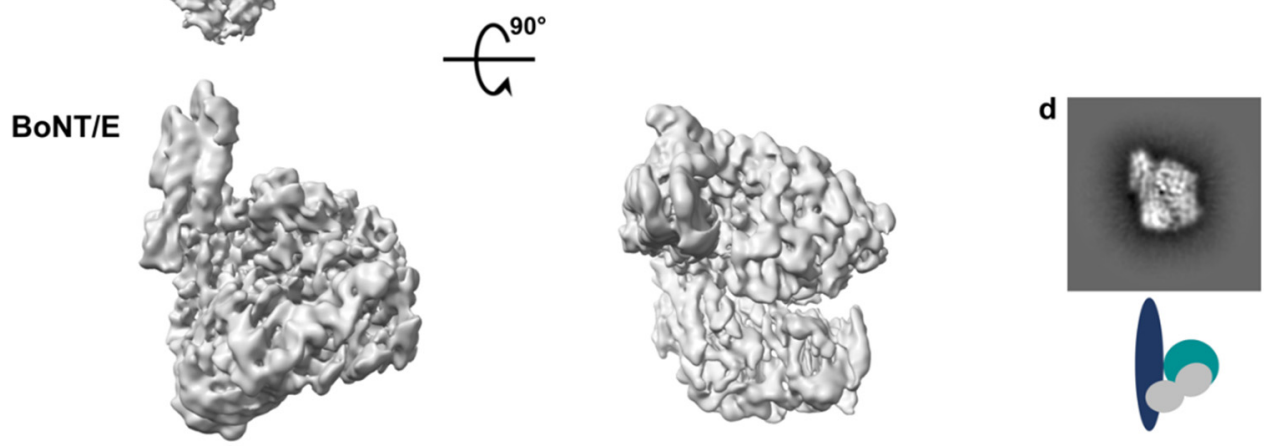

Figure 2. $(\mathbf{a}, \mathbf{b})$ Cryo-EM maps of BoNT/B and BoNT/E at an estimated resolution of 3.6 and $3.7 \AA$, respectively. The maps, as well as comparative $2 \mathrm{D}$ class averages of the toxins in $(\mathbf{c}, \mathbf{d})$, reveal clear secondary elements and domain arrangements illustrated by a schematic diagram (LC in green, $\mathrm{H}_{\mathrm{N}}$ in blue, $\mathrm{H}_{\mathrm{C}}$ in grey).

\subsubsection{Fitting of Protein Coordinates}

Cryo-EM maps of BoNT/B and /E confirmed the previously determined crystallographic structures of these holotoxins, which first revealed their different architecture $[28,29]$. Electron microscopy is often described as offering close to native structure. Here, BoNT/B also presents a linear arrangement of its three domains, whereas the BoNT/E map shows it retained its more compact domain organisation (Figure 3), thus alleviating reservation that this singular domain organisation among BoNTs may have been experimental artefacts $[29,31]$. Although the overall folds were similar, domains had to be fitted individually as rigid bodies to account for the observed local conformation variability. The domains were first docked in the cryo-EM maps with ChimeraX [36] before further real-space refinement on the whole toxins using the Phenix package [37].

The map is particularly well defined for the light chain (Supplementary Figures S1 and S2) and shows that all secondary structure elements are conserved. Despite the disorder observed for some of the larger flexible loops, the resolution obtained was sufficient to confidently assign side chains, particularly around the active site (Figure 3). Our structures are of inactive variants of the toxins presenting dual mutations at the catalytic site (BoNT/B1 $\mathrm{E} 231 \mathrm{Q} / \mathrm{H} 234 \mathrm{Y}$ and BoNT/E1 E213Q/H216Y) [38]. LC is a metalloprotease, which normally contains a catalytic $\mathrm{Zn}^{2+}$ ion coordinated by the conserved HExxH motif [39]. A similar set of mutations (i.e., HQxxY) in BoNT/A was previously shown to prevent binding of the catalytic $\mathrm{Zn}^{2+}$ ion [40], which is also not visible in either of the two maps presented here. It should, however, be noted that whilst X-ray crystallography can precisely locate and assign protein-bound metal ions using X-ray fluorescence and anomalous diffraction [41], images collected with a standard transmission electron microscope, which are based on bright-field phase contrast, do not have sufficient sensitivity to identify isolated metal atoms [42]. 
a

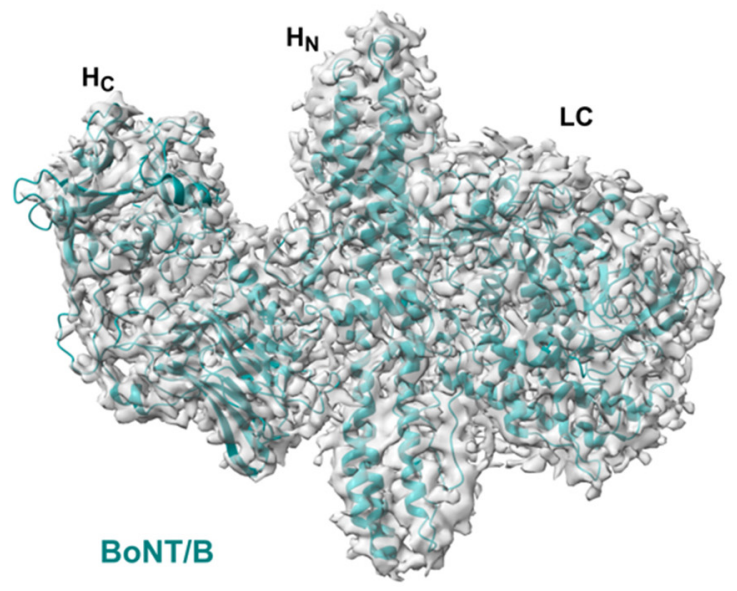

b

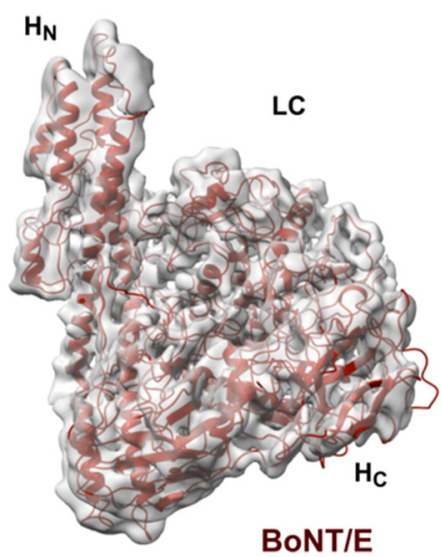

C

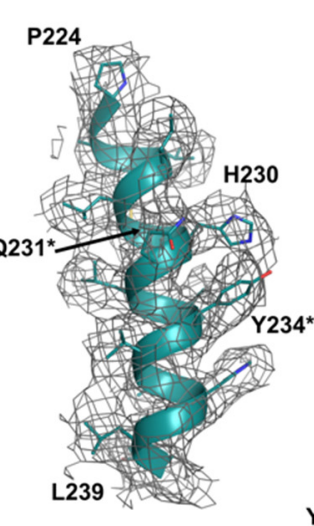

$L C$

d

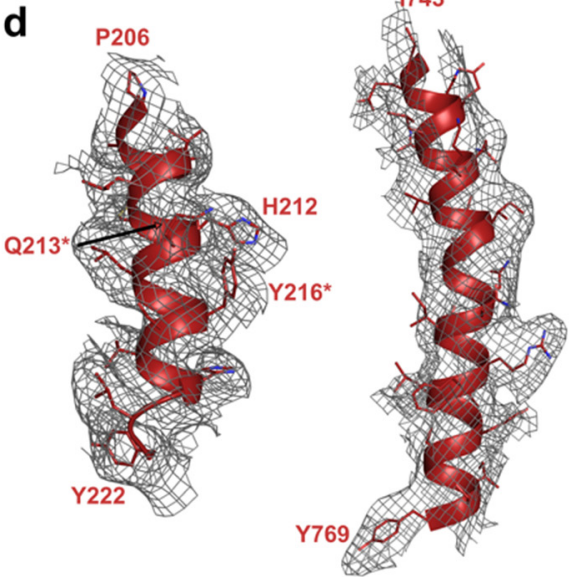

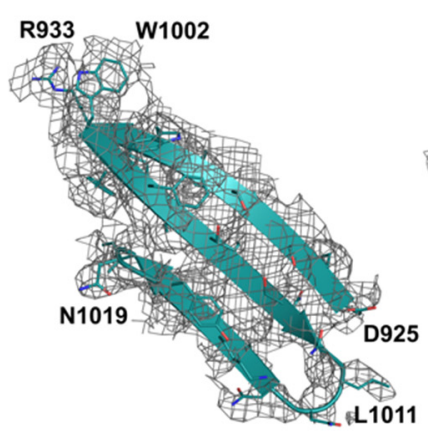

$H_{C N}$

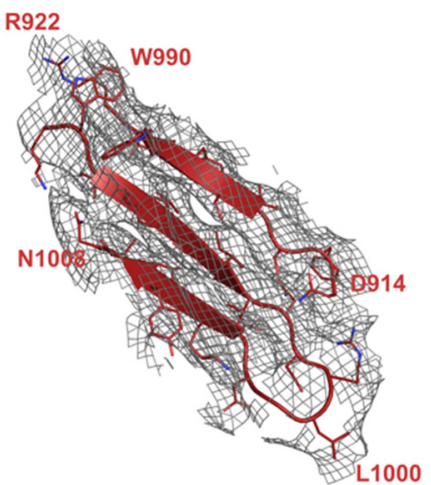

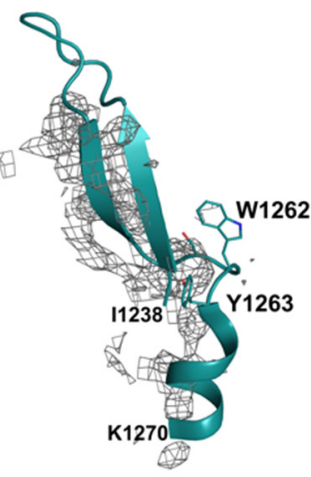

$H_{C C}$

Figure 3. $(\mathbf{a}, \mathbf{b}) 3 \mathrm{D}$ reconstruction of BoNT/B (teal) and BoNT/E (red), respectively. (c,d) Close-up map of secondary features from each domain, including the mutated catalytic site (mutations marked by *, E231Q/H234Y and E213Q/H216Y for BoNT/B and BoNT/E, respectively); $\alpha$-helix of $\mathrm{H}_{\mathrm{N}}$, $\beta$-sheet of $\mathrm{H}_{\mathrm{CN}}$, and the ganglioside-binding site of $\mathrm{H}_{\mathrm{CC}}$.

The translocation domain $\left(\mathrm{H}_{\mathrm{N}}\right)$, which represents the central feature consisting of two long (approximately $100 \AA$ ), coiled-coil helices flanked by shorter $\alpha$-helices is relatively well-defined. The resolution observed in the core region of $\mathrm{H}_{\mathrm{N}}$ provided enough details to discern side chains, although the map definition became less precise in the polar parts of the domain (Figure 3). In addition, the so called 'belt region', which is an integral part 
of $\mathrm{H}_{\mathrm{N}}$ at the primary sequence level, wraps around LC and closely interlocks the two domains together to stabilise their interaction, possibly acting as a protective chaperone for the holotoxins [43]. In the cryo-EM map of BoNT/B, the belt is well-ordered except for a small surface-exposed $\alpha$-helix (residues 487-495; Supplementary Figure S3), whereas in BoNT/E, a section could not be modelled due to the lack of map for residues 499-515, which forms a random coil in the crystal structure.

For both toxins, the position of the binding domain $\left(\mathrm{H}_{\mathrm{C}}\right)$ was clear; however, the maps could not be interpreted in as much detail (Figure 3) and should, thus, be analysed with caution. This may be due to several factors. For BoNT/B, the lack of density in some areas, particularly the $\mathrm{H}_{\mathrm{CC}}$ subdomain, suggests that the preferred particle orientation observed in the angular distribution map (Supplementary Figure S1) is the main reason affecting map quality and resolution, although local dynamism of the domain cannot be excluded. Such effect might be compensated by collection of larger datasets with altered parameters or grid preparation. However this kind of optimisation depends on a balance between the experimental goal and resource availability. Since $\mathrm{H}_{\mathrm{C}}$ / B has been well defined by $\mathrm{X}$-ray crystallography, it was fully included in the deposited coordinates associated with the map.

In the case of BoNT/E, the map around $\mathrm{H}_{\mathrm{C}}$ is also generally of lower resolution (Supplementary Figure S2), resulting in a blurrier picture. The main protein chain could be positioned, but it was difficult to carry out a stringent real-space refinement. Again, availability of the crystal structure for this domain allowed us to include it fully in our cryoEM model. Remarkably, serotype E is the only BoNT in which the domain has so far been seen in a closed configuration [3]. Furthermore, the crystal structure of the BoNT/E-NTNH (progenitor M) complex [44] had shown that $\mathrm{H}_{\mathrm{C}} / \mathrm{E}$ could take on a different position with a $60^{\circ}$ rotation relative to the other two holotoxin domains $\left(\mathrm{LC}+\mathrm{H}_{\mathrm{N}}\right)$ in a conformation stabilised by its interaction with NTNH and mediated by a flexible helical linker (BoNT/E residues 830-845) [29]. Observations from the cryo-EM map suggest $\mathrm{H}_{C} / \mathrm{E}$ is inherently dynamic thanks to the flexible linker with $\mathrm{H}_{\mathrm{N}}$, which would affect the local resolution even though it does not imply a significant conformational change like the one observed in the progenitor complex.

\subsection{Comparison with X-ray Crystal Structures}

Although the crystal structures of BoNT/B and BoNT/E were used to produce the cryo-EM models as described above, some differences were observed when comparing the output from both methods (Figure 4), with direct superposition showing root mean square deviations [45] of $1.9 \AA$ (over 1218 paired residues) and $2.0 \AA$ (over 1197 paired residues) for types B and E, respectively.

The main structural variations seem to come from overall movement of the domains with respect to each other. For example, overall superposition with BoNT/B results in a nearly perfect overlap of the translocation domains, whilst the core secondary structure of LC appears shifted by approximately $1.5 \AA$, with some of the larger loops deviating more significantly. On the other side of $\mathrm{H}_{\mathrm{N}}$, the binding domain presents a more pronounced shift of up to $2 \AA$ around the $\mathrm{H}_{\mathrm{CN}}$ lectin-like subdomain, which seems to be further accentuated at the $\mathrm{H}_{\mathrm{CC}} \beta$-trefoil fold, although the cryo-EM map was weaker in that area (Figure 3) and should not be over-interpreted. Interestingly, BoNT/B presents a small helical linker between $\mathrm{H}_{\mathrm{N}}$ and $\mathrm{H}_{\mathrm{C}}$, which is similar to the one observed in BoNT/E [29]. Although no conformational changes have been reported for BoNT/B so far from several crystal structures solved at $\mathrm{pH}$ ranging from 4.0 to 7.0 [46], presence of this linker and the slight mobility observed within the cryo-EM map suggest that the binding domain could be subject to conformational changes when part of the progenitor complex with its NTNH partner, similarly to what has been observed with serotypes E [44] and A [47].

For BoNT/E, similar observations could be made with regards to the overall domain superposition, with slight shifts in domain position observed between the cryo-EM and crystal structures. Of note, part of the belt region, located in a wide crevice between two loops of LC, seems to be particularly mobile (Supplementary Figure S3). In addition, 
a small segment (residues 461-465), which is, itself, missing from the crystal structure, was visible in the cryo-EM map, highlighting the potential complementarity of the two biophysical methods.

a

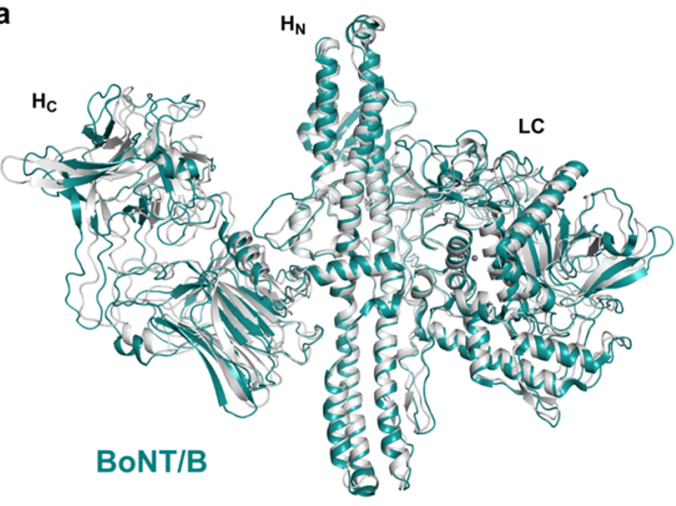

b

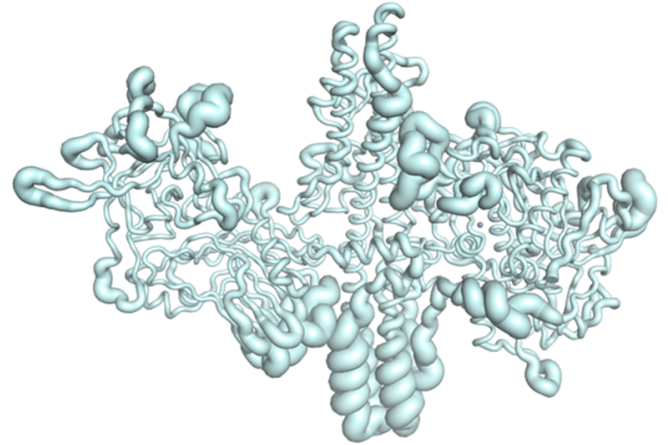

C

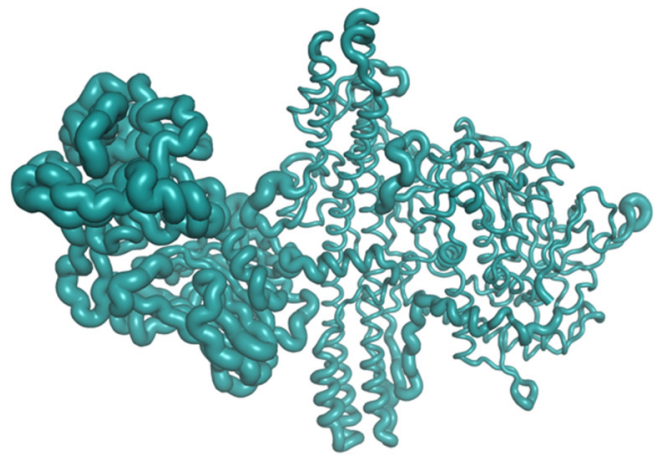

d
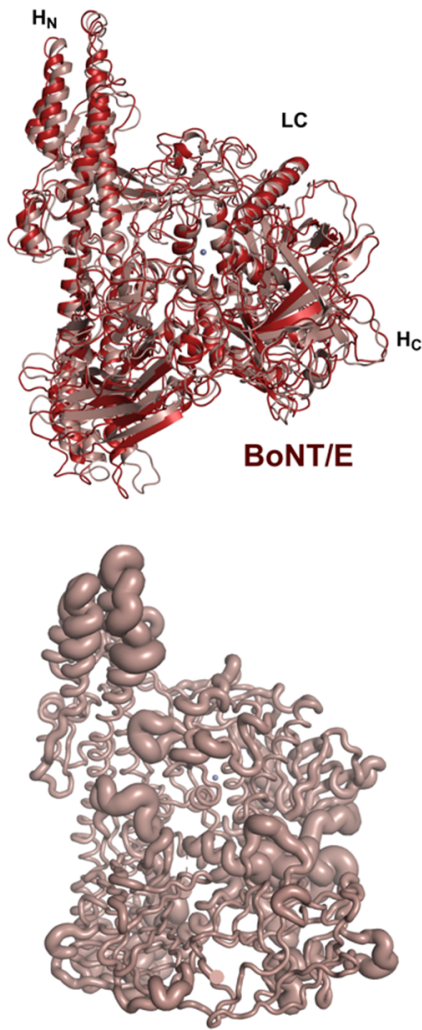

f

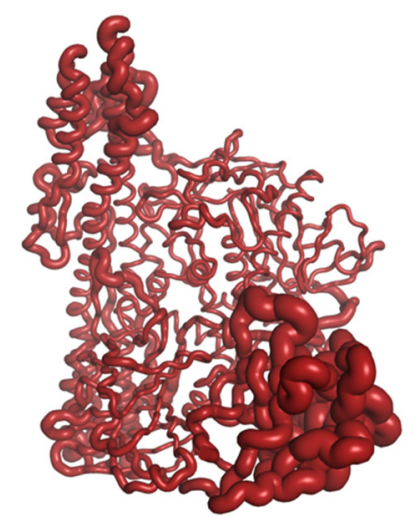

Figure 4. (a) Superposition of the cryo-EM (teal) and crystal (PDB 1EPW, light grey) coordinates of BoNT/B. (b,c) B factor-scaled ribbon representations of the crystal and cryo-EM structures of BoNT/B, respectively. (d) Superposition of the cryo-EM (red) and crystal (PDB 3FFZ, light pink) coordinates of BoNT/E. (e,f) B factor-scaled ribbon representations of the crystal and cryo-EM structures of BoNT/E, respectively.

A different approach to compare the two techniques is to analyse the resulting structures looking at their atomic B factor. The definition of the B factor term is very different between X-ray crystallography and cryo-EM; however, in each case, it provides a means to measure the disorder and, to some extent, the local dynamism in the structure, so they can be compared qualitatively [48]. Figure 4 provides an illustration of the B factors analysis for BoNT/B and BoNT/E structures emanating from the two methods, with higher B factor representing a higher state of disorder. One caveat to that analysis for crystallography is that stability of the molecules is also significantly influenced by the crystal packing and 
quality. Nonetheless, solvent-accessible loop regions typically show higher B factors, and this can be observed in $\mathrm{LC}$, the belt region, and $\mathrm{H}_{\mathrm{C}}$ domains of the crystal and cryo-EM structures for both serotypes. Remarkably, the polar extremities of the $\mathrm{H}_{\mathrm{N}}$ helices also present higher B factor in the crystal structures despite appearing clearly in the cryo-EM maps. One of the main differences resides in the binding domain, which is very well defined in the crystallographic structures but lacks resolution in the cryo-EM maps presented here. This represents a clear limitation to the method, and improvements need to be made so that atomic resolution can be reached to look at specific structural questions such as toxin-receptor or toxin-substrate interactions, which have eluded crystallography so far.

Cryo-electron microscopy has become a method of choice to determine the structure of macromolecules at near atomic resolution in close to native conditions, particularly for large and dynamic molecules for which crystallisation remains a challenge. However, cryo-EM is still limited by the size of smaller molecules and resolution obtained due to particle heterogeneity, which results in maps with high variability. Although these hurdles have hindered the obtention of high-resolution images for botulinum neurotoxins, the technique can provide a unique insight to study their molecular dynamism and catch multiple conformations that may be essential to understand the mechanism of action of BoNTs. This, for example, allowed the discovery of significant $\mathrm{pH}$-mediated conformational changes in the related tetanus toxin [32]. The recent release of a database of AlphaFold-predicted models [49] for proteins of interest included several botulinum neurotoxins. Remarkably, whilst BoNT/E retains the domain organisation described in our study, BoNT/B appears incorrectly predicted with a shift in the binding domain position not observed in any experimental data (Supplementary Figure S4). Structural predictions are valuable but should be used cautiously and concurrently with experimental methods to determine accurate protein structures.

The single particle cryo-EM maps of BoNT/B and /E presented here provide a complementary perspective on the molecular structure of these toxins. In particular, it highlights the local mobility of each domain, as well as confirms unambiguously the structural information that had been obtained by X-ray crystallography. With the recent discovery of multiple BoNT and BoNT-like proteins, cryo-EM provides a useful biophysical technique to gain essential information on the mechanism of action of this powerful toxin family. Our study provides a methodological framework to analyse the atomic structure of botulinum neurotoxins and may help the design of novel antitoxin strategies or the development of new BoNT of clinical relevance.

\section{Materials and Methods}

\subsection{Constructs}

DNA encoding nontoxic, enzymatically inactive variants of BoNT/B1 (E231Q/ H234Y) [38] (strain: Okra, residues 1 to 1291, UniProtKB: B1INP5) was kindly provided by Ipsen (Abingdon, UK) into a modified pET32a(+) vector in fusion with a C-terminal poly-His (6x) tag.

BoNT/E1 inactive variant (E213Q/H216Y) (strain: Beluga, residues 1 to 1252, UniProtKB: A8Y875) was synthesised by Genscript (Leiden, Netherlands) and cloned into a modified pET28a(+) vector designed to express the protein in fusion with a N-terminal 10xHis-tag, and TEV protease cleavage site.

\subsection{Protein Expression and Purification}

Both proteins were expressed in TB media inoculated using BL21(DE3) cells (New England Biolabs, Ipswich, MA, USA) transformed with the respective vector. Cultures were grown in a LEX bioreactor (Epiphyte Three Inc., Toronto, ON, Canada) at $37^{\circ} \mathrm{C}$. When the OD600 reached 0.8 , the temperature was reduced to $18^{\circ} \mathrm{C}$, and protein expression was induced through the addition of $1 \mathrm{mM}$ IPTG. Cells were grown for a further $18 \mathrm{~h}$ before harvesting by centrifugation. All chemical reagents were purchased from Sigma-Aldrich (Stockholm, Sweden). 
For BoNT/B, cells were resuspended in 20 mM TRIS pH 7.5, $200 \mathrm{mM} \mathrm{NaCl,} 25 \mathrm{mM}$ Imidazole and lysed using an Emulsiflex-C3 (Avestin Europe, Mannheim, Germany) at $20 \mathrm{kPsi}$. The lysate was clarified by centrifugation at $50,000 \times g$ for $45 \mathrm{~min}$ before loading onto a pre-equilibrated $5 \mathrm{~mL}$ HisTrap FF column (Cytiva, Uppsala, Sweden). Purified protein was eluted using a step elution with $20 \mathrm{mM}$ TRIS pH 7.5, $200 \mathrm{mM} \mathrm{NaCl}, 250 \mathrm{mM}$ Imidazole. Fractions containing BoNT/B1 were pooled and further purified using a Superdex200 26/600 column (Cytiva, Uppsala, Sweden) pre-equilibrated using 25 mM TRIS $\mathrm{pH}$ 7.5, $200 \mathrm{mM} \mathrm{NaCl}$, 5\% Glycerol. Protein was concentrated to $15 \mathrm{mg} / \mathrm{mL}$ with Vivaspin filters (10 kDa cut off, Sartorius, Goettingen, Germany).

For BoNT/E, cells were resuspended in 50 mM Hepes pH 7.2, $200 \mathrm{mM} \mathrm{NaCl}, 25 \mathrm{mM}$ Imidazole and lysed by sonication. The lysate was clarified by centrifugation at $42,000 \times g$ for $1 \mathrm{~h}$ before loading onto a pre-equilibrated $5 \mathrm{~mL}$ HisTrap FF column (Cytiva, Uppsala, Sweden). Purified protein was eluted using a step elution with $50 \mathrm{mM}$ Hepes $\mathrm{pH} 7.2$, $200 \mathrm{mM} \mathrm{NaCl}, 250 \mathrm{mM}$ Imidazole, and 5\% glycerol. Fractions containing BoNT/E were pooled and further purified using a Superdex200 16/600 column (Cytiva, Sweden) and pre-equilibrated using $50 \mathrm{mM}$ Hepes pH 7.2, $200 \mathrm{mM} \mathrm{NaCl}, 5 \%$ Glycerol. Protein was concentrated to $1.4 \mathrm{mg} / \mathrm{mL}$ with Vivaspin filters (100 kDa cut off, Sartorius, Goettingen, Germany).

Final concentration was measured by absorbance at $280 \mathrm{~nm}$ (NanoDrop Spectrophotometer, Thermo Fisher Scientific, Göteborg, Sweden), and proteins were flash frozen in liquid nitrogen for storage at $-80^{\circ} \mathrm{C}$ until further use.

\subsection{Single Particle Cryo-Electron Microscopy}

\subsubsection{Cryo-EM Grid Preparation}

BoNT/B was prepared with an additional size exclusion chromatography step using a Superdex200 10/300 column (Cytiva, Uppsala, Sweden) pre-equilibrated in 20 mM HEPES pH 7.5, $200 \mathrm{mM} \mathrm{NaCl}, 0.5 \mathrm{mM}$ TCEP. Sample at $0.05 \mathrm{mg} / \mathrm{mL}$ in $20 \mathrm{mM}$ HEPES pH 7.5, $50 \mathrm{mM} \mathrm{NaCl}$ was pipetted onto glow-discharged holey carbon cryo-EM grids (Quantifoil $\mathrm{Cu}$ R0.6/1) and frozen in liquid ethane using a Vitrobot (Thermo Fisher Scientific, Göteborg, Sweden).

BoNT/E was prepared with an additional size exclusion chromatography step using a Superdex200 10/300 column (Cytiva, Uppsala, Sweden) pre-equilibrated in $20 \mathrm{mM}$ HEPES pH 7.2, $200 \mathrm{mM} \mathrm{NaCl}, 1 \mathrm{mM}$ TCEP. Sample at $0.1 \mathrm{mg} / \mathrm{mL}$ in $20 \mathrm{mM}$ HEPES pH 7.5, $50 \mathrm{mM} \mathrm{NaCl}$ was pipetted onto glow-discharged holey carbon cryo-EM grids (Cu R1.2/1.3, Quantifoil, Großlöbichau, Germany) and frozen in liquid ethane using a Vitrobot (Thermo Fisher Scientific, Göteborg, Sweden).

\subsubsection{Cryo-EM Imaging}

Cryo-EM experiments were conducted at the Swedish Cryo-EM National Facility, Umeå and Stockholm nodes (Sweden). Movies were collected in a Titan Krios (Thermo Fisher Scientific, Göteborg, Sweden) microscope operating at $300 \mathrm{kV}$ in electron-counting mode and at a nominal magnification of $130,000 \times(1.09 \AA / p x)$ and $165,000 \times(0.86 \AA / p x)$ with an electron flux of $57.3 \mathrm{e} / \AA^{2}$ and $55 \mathrm{e} / \AA^{2}$ over 40 frames for BoNT/B and BoNT/E, respectively. The data were recorded using a Gatan K2 Summit (AMETEK, Leicester, UK) direct detector coupled with a Bioquantum energy filter, with a $20 \mathrm{eV}$ slit.

\subsubsection{Cryo-EM Data Processing}

For BoNT / B the frames were aligned, averaged, and dose-weighted in cryoSPARC [50]. CTF estimation and downstream processing were also carried out in cryoSPARC. A total of 2252 movies were recorded, from which a total of 2,432,309 particles were automatically picked, and after 2D classification, 676,677 particles were selected and used for 3D refinement, yielding the final map at 3.6 ̊ resolution, calculated based on the gold standard FSC of 0.143 . The density map was sharpened by applying a negative B factor. 
For BoNT / E the frames were aligned, averaged, and dose-weighted in cryoSPARC [50]. CTF estimation and downstream processing were also carried out in cryoSPARC. A total of 18,282 movies were recorded, from which a total of 1,096,741 particles were automatically picked, and after 2D classification, 284,390 particles were selected and used for 3D refinement, yielding the final map at $3.7 \AA$ resolution, calculated based on the gold standard FSC of 0.143 . The density map was sharpened by applying a negative B factor.

\subsubsection{Model Building and Validation}

Protein validation was performed with MolProbity [51]. Data statistics are summarized in Table 2. The atomic coordinates and cryo-EM maps were deposited in the Protein Data Bank (PDB) with ID 7QFQ and 7QFP and the Electron Microscopy Data Base (EMDB) with ID 13947 and 13946 for BoNT/B and BoNT/E, respectively. Protein structure figures were rendered with PyMOL (Schrödinger, LLC, New York, NY, USA) or ChimeraX [36].

Table 2. Cryo-EM Data Collection and Refinement.

\begin{tabular}{|c|c|c|}
\hline & BoNT/B & BoNT/E \\
\hline \multicolumn{3}{|l|}{ Data collection and processing } \\
\hline Nominal magnification & 130,000 & 165,000 \\
\hline Voltage (kV) & 300 & 300 \\
\hline Electron exposure $\left(\mathrm{e} / \AA^{2}\right)$ & 57.3 & 55 \\
\hline Defocus range $(\mu \mathrm{m})$ & $-1.9--3.5$ & $-0.5--3$ \\
\hline Pixel size $(\AA)$ & 1.09 & 0.86 \\
\hline Number of images & 2252 & 18,282 \\
\hline Symmetry imposed & $\mathrm{C} 1$ & C1 \\
\hline Particles picked & $2,420,309$ & $1,096,741$ \\
\hline Particles refined & 286,802 & 284,390 \\
\hline Map resolution $(\AA)$ & 3.6 & 3.7 \\
\hline FSC threshold & 0.143 & 0.143 \\
\hline Map sharpening B factor & -151.9 & -100 \\
\hline \multicolumn{3}{|l|}{ Refinement } \\
\hline Initial model used (PDB code) & $1 \mathrm{EPW}$ & 3FFZ \\
\hline \multicolumn{3}{|l|}{ Model composition } \\
\hline Nonhydrogen atoms & 10,660 & 9996 \\
\hline Protein residues & 1291 & 1233 \\
\hline Ligand & 0 & 0 \\
\hline B factors $\left(\AA^{2}\right)$ & 160 & 54 \\
\hline R.m.s.d. Bond lengths ( & 0.002 & 0.007 \\
\hline R.m.s.d. Bond angles $\left(^{\circ}\right)$ & 0.552 & 1.118 \\
\hline \multicolumn{3}{|l|}{ Validation } \\
\hline MolProbity score & 1.82 & 2.82 \\
\hline Clash score & 8.21 & 18.19 \\
\hline Poor rotamers $(\%)$ & 0 & 6.2 \\
\hline \multicolumn{3}{|l|}{ Ramachandran statistics: } \\
\hline Favoured (\%) & 94.5 & 86.6 \\
\hline Outliers (\%) & 0 & 0.5 \\
\hline PDB/EMDB ID & 7QFQ/13947 & 7QFP / 13946 \\
\hline
\end{tabular}

Supplementary Materials: The following are available online at https://www.mdpi.com/article/10 .3390/toxins14010014/s1: Figure S1: Image processing and evaluation of BoNT/B dataset; Figure S2: Image processing and evaluation of BoNT/E dataset; Figure S3: Belt region cryo-EM map. Figure S4: AlphaFold model predictions.

Author Contributions: Conceptualization, P.S. and G.M.; methodology, S.K., M.M.-C., J.R.D. and G.M.; validation, S.K., M.M.-C. and G.M.; writing-original draft preparation, S.K., M.M.-C. and G.M.; final writing, G.M.; review and editing, G.M. and P.S.; supervision, P.S. All authors have read and agreed to the published version of the manuscript. 
Funding: This work was supported by grants from the Novo Nordisk Foundation (NNF20OC0064789), the Swedish Research Council (2018-03406) and the Swedish Cancer Society (20 1287 PjF) to P.S. G.M. was supported by a Research Fellowship from Applied Molecular Transport Inc. (San Francisco, CA, USA) at the University of Bath (UK). The data was collected at the Cryo-EM Swedish National Facility funded by the Knut and Alice Wallenberg, Family Erling Persson and Kempe Foundations, SciLifeLab, Stockholm University and Umeå University.

Data Availability Statement: The atomic coordinates and cryo-EM maps have been deposited in the Protein Data Bank (PDB, https: / / www.rcsb.org/, accessed on 5 December 2021) and the Electron Microscopy Data Base (EMDB, https:/ / www.ebi.ac.uk/emdb/, accessed on 5 December 2021).

Acknowledgments: The authors would like to thank scientists at the Cryo-EM Swedish National Facility (SciLifeLab in Stockholm, and Umeå Core Facility for Electron Microscopy at Umeå University) for support during data collection.

Conflicts of Interest: The authors declare no conflict of interest. The funders had no role in the design of the study; in the collection, analyses, nor interpretation of data; in the writing of the manuscript; nor in the decision to publish the results.

\section{References}

1. Williamson, C.H.; Sahl, J.W.; Smith, T.J.; Xie, G.; Foley, B.T.; Smith, L.A.; Fernández, R.A.; Lindström, M.; Korkeala, H.; Keim, P.; et al. Comparative genomic analyses reveal broad diversity in botulinum-toxin-producing Clostridia. BMC Genom. 2016, 17, 180. [CrossRef] [PubMed]

2. Zhang, S.; Masuyer, G.; Zhang, J.; Shen, Y.; Lundin, D.; Henriksson, L.; Miyashita, S.I.; Martinez-Carranza, M.; Dong, M.; Stenmark, P. Identification and characterization of a novel botulinum neurotoxin. Nat. Commun. 2017, 8, 14130. [CrossRef]

3. Dong, M.; Masuyer, G.; Stenmark, P. Botulinum and tetanus neurotoxins. Annu. Rev. Biochem. 2019, 88, 811-837. [CrossRef] [PubMed]

4. Rossetto, O.; Montecucco, C. Tables of toxicity of botulinum and tetanus neurotoxins. Toxins 2019, 11, 686. [CrossRef] [PubMed]

5. Rasetti-Escargueil, C.; Popoff, M.R. Antibodies and vaccines against botulinum toxins: Available measures and novel approaches. Toxins 2019, 11, 528. [CrossRef]

6. Stenmark, P.; Dupuy, J.; Imamura, A.; Kiso, M.; Stevens, R.C. Crystal structure of botulinum neurotoxin type A in complex with the cell surface co-receptor GT1b-insight into the toxin-neuron interaction. PLoS Pathog. 2008, 4, e1000129. [CrossRef]

7. Berntsson, R.P.A.A.; Peng, L.; Dong, M.; Stenmark, P. Structure of dual receptor binding to botulinum neurotoxin B. Nat. Commun. 2013, 4, 2058. [CrossRef]

8. Masuyer, G.; Davies, J.R.; Stenmark, P. Mechanism of ganglioside receptor recognition by botulinum neurotoxin serotype E. Int. J. Mol. Sci. 2021, 22, 8315. [CrossRef]

9. Binz, T.; Rummel, A. Cell entry strategy of clostridial neurotoxins. J. Neurochem. 2009, 109, 1584-1595. [CrossRef]

10. Nishiki, T.; Kamata, Y.; Nemoto, Y.; Omori, A.; Ito, T.; Takahashi, M.; Kozaki, S. Identification of protein receptor for Clostridium botulinum type B neurotoxin in rat brain synaptosomes. J. Biol. Chem. 1994, 269, 10498-10503. [CrossRef]

11. Nishiki, T.; Tokuyama, Y.; Kamata, Y.; Nemoto, Y.; Yoshida, A.; Sato, K.; Sekiguchi, M.; Takahashi, M.; Kozaki, S. The high-affinity binding of Clostridium botulinum type B neurotoxin to synaptotagmin II associated with gangliosides GT1b/GD1a. FEBS Lett. 1996, 378, 253-257. [CrossRef]

12. Dong, M.; Richards, D.A.; Goodnough, M.C.; Tepp, W.H.; Johnson, E.A.; Chapman, E.R. Synaptotagmins I and II mediate entry of botulinum neurotoxin B into cells. J. Cell Biol. 2003, 162, 1293-1303. [CrossRef] [PubMed]

13. Rummel, A.; Karnath, T.; Henke, T.; Bigalke, H.; Binz, T. Synaptotagmins I and II act as nerve cell receptors for botulinum neurotoxin G. J. Biol. Chem. 2004, 279, 30865-30870. [CrossRef] [PubMed]

14. Jin, R.; Rummel, A.; Binz, T.; Brunger, A.T. Botulinum neurotoxin B recognizes its protein receptor with high affinity and specificity. Nature 2006, 444, 1092-1095. [CrossRef]

15. Chai, Q.; Arndt, J.W.; Dong, M.; Tepp, W.H.; Johnson, E.A.; Chapman, E.R.; Stevens, R.C. Structural basis of cell surface receptor recognition by botulinum neurotoxin B. Nature 2006, 444, 1096-1100. [CrossRef]

16. Dong, M.; Tepp, W.H.; Liu, H.; Johnson, E.A.; Chapman, E.R. Mechanism of botulinum neurotoxin B and G entry into hippocampal neurons. J. Cell Biol. 2007, 179, 1511-1522. [CrossRef] [PubMed]

17. Peng, L.; Berntsson, R.P.; Tepp, W.H.; Pitkin, R.M.; Johnson, E.A.; Stenmark, P.; Dong, M. Botulinum neurotoxin D-C uses synaptotagmin I and II as receptors, and human synaptotagmin II is not an effective receptor for type B, D-C and G toxins. J. Cell Sci. 2012, 125, 3233-3242. [CrossRef] [PubMed]

18. Montal, M. Botulinum neurotoxin: A marvel of protein design. Ann. Rev. Biochem. 2010, 79, 591-617. [CrossRef]

19. Südhof, T.C.; Rothman, J.E. Membrane fusion: Grappling with SNARE and SM proteins. Science 2009, 323, 474-477. [CrossRef]

20. Schiavo, G.; Matteoli, M.; Montecucco, C. Neurotoxins affecting neuroexocytosis. Physiol. Rev. 2000, 80, 717-766. [CrossRef]

21. Fonfria, E.; Maignel, J.; Lezmi, S.; Martin, V.; Splevins, A.; Shubber, S.; Kalinichev, M.; Foster, K.; Picaut, P.; Krupp, J. The expanding therapeutic utility of botulinum neurotoxins. Toxins 2018, 10, 208. [CrossRef] [PubMed] 
22. Steward, L.; Brin, M.F.; Brideau-Andersen, A. Novel native and engineered botulinum neurotoxins. Handb. Exp. Pharmacol. 2021, 263, 63-89. [CrossRef] [PubMed]

23. Donald, S.; Elliott, M.; Gray, B.; Hornby, F.; Lewandowska, A.; Marlin, S.; Favre-Guilmard, C.; Périer, C.; Cornet, S.; Kalinichev, M.; et al. A comparison of biological activity of commercially available purified native botulinum neurotoxin serotypes A1 to F1 in vitro, ex vivo, and in vivo. Pharmacol. Res. Perspect. 2018, 6, e00446. [CrossRef] [PubMed]

24. Eleopra, R.; Rinaldo, S.; Montecucco, C.; Rossetto, O.; Devigili, G. Clinical duration of action of different botulinum toxin types in humans. Toxicon 2020, 179, 84-91. [CrossRef] [PubMed]

25. Pons, L.; Vilain, C.; Volteau, M.; Picaut, P. Safety and pharmacodynamics of a novel recombinant botulinum toxin E (rBoNT-E): Results of a phase 1 study in healthy male subjects compared with abobotulinumtoxinA (Dysport ${ }^{\circledR}$ ). J. Neurol. Sci. 2019, 407, 116516. [CrossRef]

26. Yoelin, S.G.; Dhawan, S.S.; Vitarella, D.; Ahmad, W.; Hasan, F.; Abushakra, S. Safety and efficacy of EB-001, a novel type E botulinum toxin, in subjects with glabellar frown lines: Results of a phase 2, Randomized, Placebo-Controlled, Ascending-Dose Study. Plast. Reconstr. Surg. 2018, 142, 847e-855e. [CrossRef] [PubMed]

27. Lacy, D.B.; Tepp, W.; Cohen, A.C.; DasGupta, B.R.; Stevens, R.C. Crystal structure of botulinum neurotoxin type A and implications for toxicity. Nat. Struct. Biol. 1998, 5, 898-902. [CrossRef]

28. Swaminathan, S.; Eswaramoorthy, S. Structural analysis of the catalytic and binding sites of Clostridium botulinum neurotoxin B. Nat. Struct. Biol. 2000, 7, 693-699. [CrossRef]

29. Kumaran, D.; Eswaramoorthy, S.; Furey, W.; Navaza, J.; Sax, M.; Swaminathan, S. Domain organization in Clostridium botulinum neurotoxin type $\mathrm{E}$ is unique: Its implication in faster translocation. J. Mol. Biol. 2009, 386, 233-245. [CrossRef] [PubMed]

30. Wang, J.; Meng, J.; Lawrence, G.W.; Zurawski, T.H.; Sasse, A.; Bodeker, M.O.; Gilmore, M.A.; Fernández-Salas, E.; Francis, J.; Steward, L.E.; et al. Novel chimeras of botulinum neurotoxins A and E unveil contributions from the binding, translocation, and protease domains to their functional characteristics. J. Biol. Chem. 2008, 283, 16993-17002. [CrossRef]

31. Fischer, A.; Garcia-Rodriguez, C.; Geren, I.; Lou, J.; Marks, J.D.; Nakagawa, T.; Montal, M. Molecular architecture of botulinum neurotoxin E revealed by single particle electron microscopy. J. Biol. Chem. 2008, 283, 3997-4003. [CrossRef]

32. Masuyer, G.; Conrad, J.; Stenmark, P. The structure of the tetanus toxin reveals pH-mediated domain dynamics. EMBO Rep. 2017, 18, 1306-1317. [CrossRef] [PubMed]

33. Tamura, K.; Stecher, G.; Kumar, S. MEGA11: Molecular evolutionary genetics analysis version 11. Mol. Biol. Evol. 2021, 38 , 3022-3027. [CrossRef] [PubMed]

34. Madeira, F.; Park, Y.M.; Lee, J.; Buso, N.; Gur, T.; Madhusoodanan, N.; Basutkar, P.; Tivey, A.R.N.; Potter, S.C.; Finn, R.D.; et al The EMBL-EBI search and sequence analysis tools APIs in 2019. Nucleic Acids Res. 2019, 47, W636-W641. [CrossRef] [PubMed]

35. Chaddock, J.A.; Herbert, M.H.; Ling, R.J.; Alexander, F.C.; Fooks, S.J.; Revell, D.F.; Quinn, C.P.; Shone, C.C.; Foster, K.A. Expression and purification of catalytically active, non-toxic endopeptidase derivatives of Clostridium botulinum toxin type A. Protein Expr. Purif. 2002, 25, 219-228. [CrossRef]

36. Pettersen, E.F.; Goddard, T.D.; Huang, C.C.; Meng, E.C.; Couch, G.S.; Croll, T.I.; Morris, J.H.; Ferrin, T.E. UCSF ChimeraX: Structure visualization for researchers, educators, and developers. Protein Sci. 2021, 30, 70-82. [CrossRef] [PubMed]

37. Liebschner, D.; Afonine, P.V.; Baker, M.L.; Bunkóczi, G.; Chen, V.B.; Croll, T.I.; Hintze, B.; Hung, L.W.; Jain, S.; McCoy, A.J.; et al. Macromolecular structure determination using X-rays, neutrons and electrons: Recent developments in Phenix. Acta Crystallogr. D Struct. Biol. 2019, 75, 861-877. [CrossRef] [PubMed]

38. Stancombe, P.R.; Masuyer, G.; Birch-Machin, I.; Beard, M.; Foster, K.A.; Chaddock, J.A.; Acharya, K.R. Engineering botulinum neurotoxin domains for activation by toxin light chain. FEBS J. 2012, 279, 515-523. [CrossRef]

39. Breidenbach, M.A.; Brunger, A.T. Substrate recognition strategy for botulinum neurotoxin serotype A. Nature 2004, 432, 925-929. [CrossRef]

40. Masuyer, G.; Stancombe, P.; Chaddock, J.A.; Acharya, K.R. Structures of engineered Clostridium botulinum neurotoxin derivatives Acta Cryst. Sect. F Struct. Biol. Cryst. Commun. 2011, 67, 1466-1472. [CrossRef]

41. Handing, K.B.; Niedzialkowska, E.; Shabalin, I.G.; Kuhn, M.L.; Zheng, H.; Minor, W. Characterizing metal-binding sites in proteins with X-ray crystallography. Nat. Protoc. 2018, 13, 1062-1090. [CrossRef]

42. Elad, N.; Bellapadrona, G.; Houben, L.; Sagi, I.; Elbaum, M. Detection of isolated protein-bound metal ions by single-particle cryo-STEM. Proc. Natl. Acad. Sci. USA 2017, 114, 11139-11144. [CrossRef] [PubMed]

43. Brunger, A.T.; Breidenbach, M.A.; Jin, R.; Fischer, A.; Santos, J.S.; Montal, M. Botulinum neurotoxin heavy chain belt as an intramolecular chaperone for the light chain. PLoS Pathog. 2007, 3, 1191-1194. [CrossRef] [PubMed]

44. Eswaramoorthy, S.; Sun, J.; Li, H.; Singh, B.R.; Swaminathan, S. Molecular Assembly of Clostridium botulinum progenitor M complex of type E. Sci. Rep. 2015, 5, 17795. [CrossRef]

45. Krissinel, E. Enhanced fold recognition using efficient short fragment clustering. J. Mol. Biochem. 2012, 1, 76-85.

46. Eswaramoorthy, S.; Kumaran, D.; Keller, J.; Swaminathan, S. Role of metals in the biological activity of Clostridium botulinum neurotoxins. Biochemistry 2004, 43, 2209-2216. [CrossRef]

47. Gu, S.; Rumpel, S.; Zhou, J.; Strotmeier, J.; Bigalke, H.; Perry, K.; Shoemaker, C.B.; Rummel, A.; Jin, R. Botulinum neurotoxin is shielded by NTNHA in an interlocked complex. Science 2012, 335, 977-981. [CrossRef] 
48. Kaur, S.; Gomez-Blanco, J.; Khalifa, A.A.Z.; Adinarayanan, S.; Sanchez-Garcia, R.; Wrapp, D.; McLellan, J.S.; Bui, K.H.; Vargas, J. Local computational methods to improve the interpretability and analysis of cryo-EM maps. Nat. Commun. 2021, 12, 1240. [CrossRef] [PubMed]

49. Jumper, J.; Evans, R.; Pritzel, A.; Green, T.; Figurnov, M.; Ronneberger, O.; Tunyasuvunakool, K.; Bates, R.; Žídek, A.; Potapenko, A.; et al. Highly accurate protein structure prediction with AlphaFold. Nature 2021, 596, 583-589. [CrossRef]

50. Punjani, A.; Rubinstein, J.L.; Fleet, D.J.; Brubaker, M.A. cryoSPARC: Algorithms for rapid unsupervised cryo-EM structure determination. Nat. Methods 2017, 14, 290-296. [CrossRef]

51. Chen, V.B.; Arendall, W.B., 3rd; Headd, J.J.; Keedy, D.A.; Immormino, R.M.; Kapral, G.J.; Murray, L.W.; Richardson, J.S.; Richardson, D.C. MolProbity: All-atom structure validation for macromolecular crystallography. Acta Crystallogr. D 2010, 66, 12-21. [CrossRef] [PubMed] 